\title{
Identification of Eggplant mottled dwarf virus in PEQ Hibiscus syriacus plants imported from Australia
}

\author{
J. Tang $\cdot$ C. Elliott $\cdot$ L. I. Ward $\cdot$ A. Iqram
}

Received: 6 October 2014 / Accepted: 19 November 2014 / Published online: 30 November 2014

(C) Australasian Plant Pathology Society Inc. 2015

\begin{abstract}
In March 2014, leaf chlorotic spots were observed on several plants of Hibiscus syriacus which had been imported from Australia and held in post-entry quarantine (PEQ) in Auckland, New Zealand. Eggplant mottled dwarf virus (EMDV) was identified on the diseased hibiscus, using herbaceous indexing, electron microscopy, serological and molecular techniques. This appears to be the first report of EMDV presence in Australia.
\end{abstract}

Keywords Eggplant mottled dwarf virus · Hibiscus · First report · Australia

Eggplant mottled dwarf virus (EMDV) is a distinct species of the genus Nucleorhabdovirus in the family Rhabdoviridae. After the first identification in eggplant (Solanum melongena) from southern Italy in 1969 (Martelli 1969), the virus has since been reported in some other economically important crops such as cucumber, pepper, potato, tomato and tobacco and has caused quality and yield loss (Roggero et al. 1995; Babaie and Izadpanah 2002; Martelli et al. 2011). The natural hosts of EMDV also includes ornamentals (honeysuckle, pelargonium and pittosporum) and wild species (Capparis spinosa, Solanum nigrum, S. sodomaeum) (Martelli et al. 2011). The infection of EMDV in Hibiscus rosa-sinensis has been reported in Italy (De Stradis et al. 2008) and Spain (Parrella et al. 2013), although it is thought that the rhabdovirus found in H. rosa-sinensis from Greece and Spain in 1984

J. Tang $(\bowtie) \cdot$ C. Elliott $\cdot$ L. I. Ward

Plant Health and Environment Laboratory, Ministry for Primary

Industries, P.O. Box 2095, Auckland 1140, New Zealand

e-mail: joe.tang@mpi.govt.nz

A. Iqram

Verification Services, Ministry for Primary Industries,

P.O. Box 53066, Auckland 2150, New Zealand and from Morocco in 1987, was also an isolate of EMDV (Plavsic and Milicic 1984; Parrella et al. 2013).

In March 2014, chlorotic spots were observed on leaves of several plants of $H$. syriacus which had been imported from Australia and held in a post-entry quarantine (PEQ) facility in Auckland, New Zealand (Fig. 1a). Leaf tissue of symptomatic H. syriacus was mechanically inoculated onto a range of herbaceous species using the method described previously (Tang et al. 2013). The inoculated Nicotiana occidentalis cv 37B developed chlorotic/necrotic local lesions and systemic vein clearing after 18 days (Fig. 1d), followed by leaf distortion and plant stunting (Fig. 1e). N. benthamiana, $N$. clevelandii, N. glutinosa and $N$. sylvestris produced similar symptoms while Chenopodium amaranticolor and Gomphrena globosa exhibited systemic vein chlorosis and local necrotic lesions, respectively (Fig. 1b, c). No symptoms were observed on inoculated C. quinoa, Cucumis sativus and Phaseolus vulgaris. With the exception of $C$. amaranticolor, which reportedly does not produce reaction, these symptoms were similar to those described for EMDV (De Stradis et al. 2008; Martelli et al. 2011).

Transmission electron microscopy (TEM) was carried out for the original $H$. syriacus and symptomatic $N$. occidentalis and $N$. clevelandii, using a crude sap preparation as described by Tang et al. 2013. Bacilliform shaped particles approximately $250 \times 70 \mathrm{~nm}$ in size (Fig. 2) were observed from the symptomatic herbaceous species. The particles were similar in morphology and dimensions to those of EMDV previously reported in H. rosa-sinensis (De Stradis et al. 2008). No virus particles could be found in the original host.

Serological identification was performed using double antibody sandwich (DAS)-ELISA with commercial polyclonal antiserum raised against EMDV (Loewe Biochemica $\mathrm{GmbH}$ ). Leaf tissue from all symptomatic indicator plants were tested for the presence of EMDV. They were all positive while no reactions were observed for healthy controls of each species. 
Fig. 1 Foliar symptoms observed on the original Hibiscus syriacus with chlorotic spots (a) and inoculated Chenopodium amaranticolor with systemic vein chlorosis (b), Gomphrena globosa with necrotic local lesions (c), Nicotiana occidentalis with necrotic local spots and systemic vein clearing after 18 days of inoculation (d), followed by leaf distortion and plant stunting (e).

$N$. benthamiana, $N$. clevelandii, $N$. glutinosa and $N$. sylvestris produced similar symptoms as $N$. occidentalis
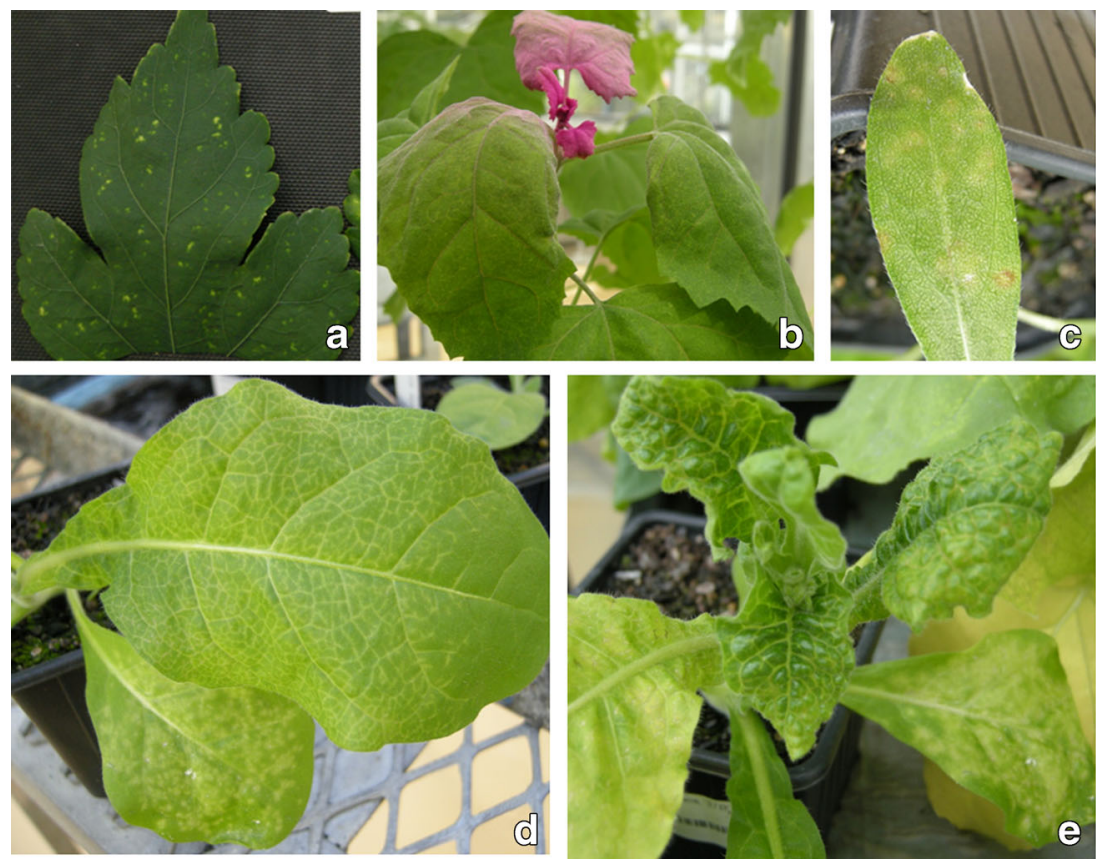

Reverse transcription (RT)-PCR was subsequently conducted to further confirm the identification of EMDV. Forward (5'- GATACCCACCTCCTGGAACAT-3') and reverse (5'-TCGGTGGAGGTGACATACA-3') primers that used in the PCR were designed on the basis of an alignment of 12 published EMDV sequences containing $\mathrm{RdRp}$ region from GenBank. A product of the expected size (309 bp) was amplified from nucleic acid extracted from the original H.syriacus and all symptomatic herbaceous species, as well as asymptomatic $C$. quinoa. The amplicons were sequenced in both directions and the consensus sequence was deposited at GenBank under the accession number of KJ742827.

In order to estimate the relationship of the EMDV isolates from Australia and from elsewhere in the world, a phylogenetic analysis was carried out using Geneious 7.1.5 software (Biomatters Ltd, Auckland, New Zealand). The phylogenetic tree (Fig. 3) constructed from the 309 bp RdRp sequences indicates that the Australia isolate is distinct from those already published in GenBank (including 6 isolates from Greece and 3 isolates each from Italy and Spain, at time of writing), which shares only $84.8-89.0 \%$ nucleotide identity to these European isolates. Moreover, the diversity of EMDV sequences seems more geography-dependent rather than host species-dependent. For example, except the Capparis isolate, EMDV sequences isolated from different host species in Greece share over $96 \%$ nucleotide identity to each other, but Pittosporum isolate from Greece only has $89-90 \%$ nucleotide identity with Pittosporum isolates from Spain. Hence no correlations between geographical origins of these EMDV isolates could be found and as such, the relationship between isolates from Australia and from elsewhere in the world is not clear.

EMDV causes vein yellowing or clearing on several host species, including Hibiscus rosa-sinensis (Lockhart 1987; De
Fig. 2 Rhabdovirus-like particles observed on Nicotiana occidentalis inoculated from Eggplant mottled dwarf virusinfected Hibiscus syriacus

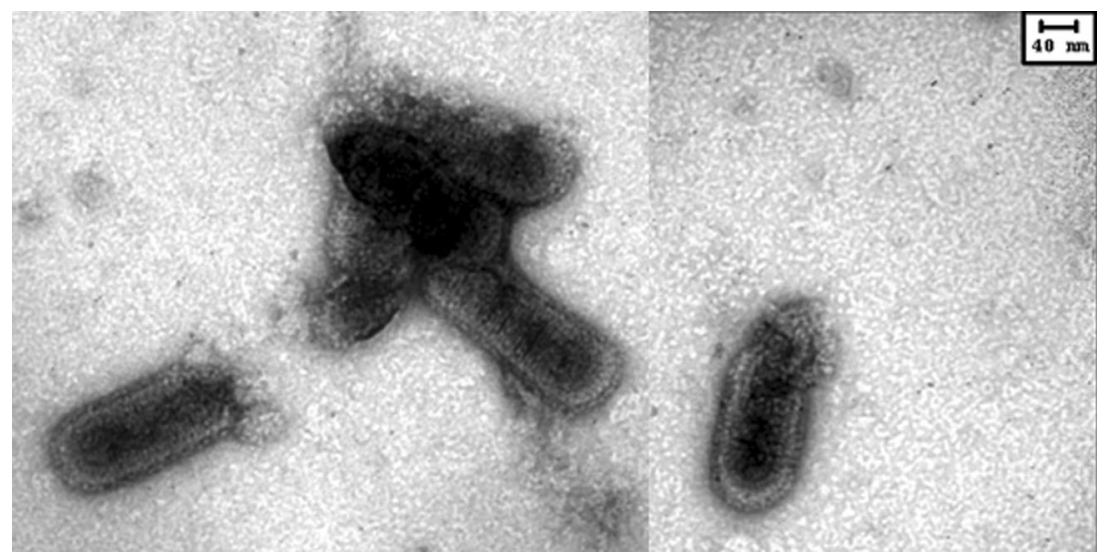




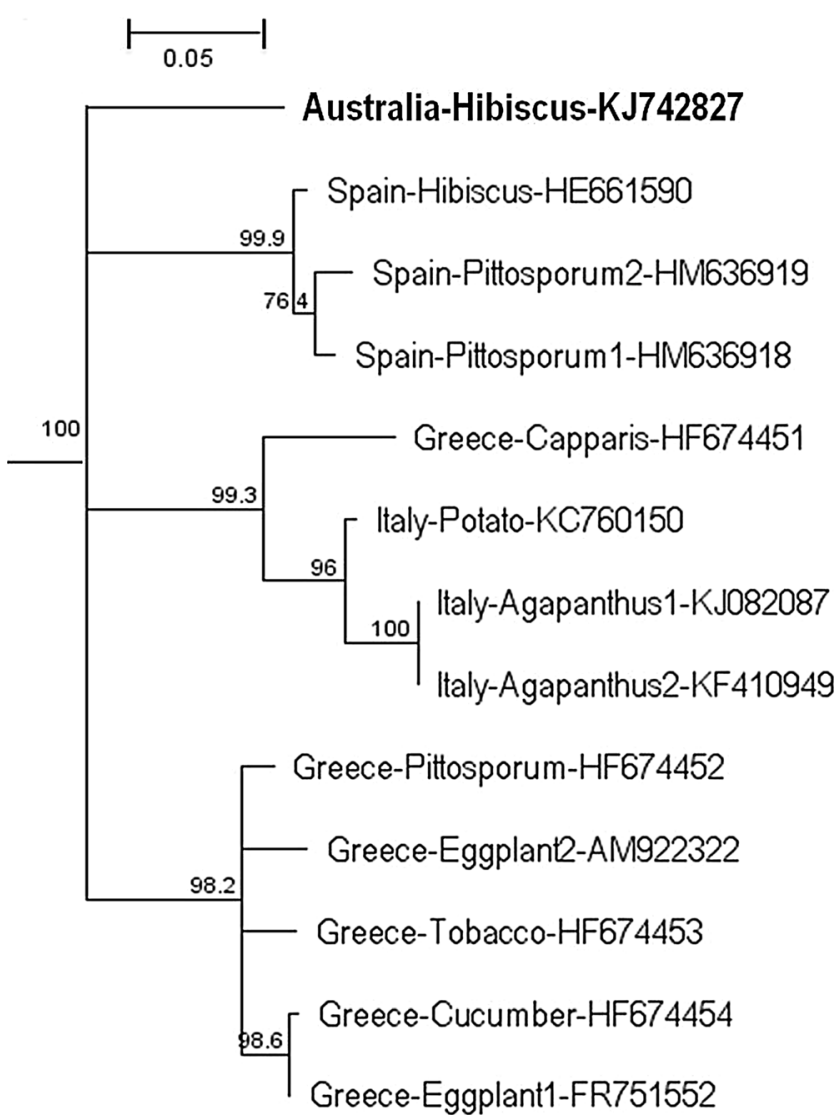

Fig. 3 Phylogenetic relationship of Eggplant mottled dwarf virus based on nucleotide sequence (309 bp) from the RNA-dependent RNA polymerase region. The format of each label is Country-Host genusGenBank accession number. Branches with more than $70 \%$ bootstrap support with 1000 replicates are shown

Stradis et al. 2008; Parrella et al. 2013), however, the EMDVinfected $H$. syriacus in this study showed chlorotic spots instead. Also, this EMDV isolate induced systemic symptoms on C. amaranticolor which differs from those reported previously. In order to rule out whether the observed symptoms was caused by a synergistic interaction of double infections, the following viruses, which have been reported to infect the genus of Hibiscus, were tested by RT-PCR using published or novel primers (data not shown) and tests for all these viruses were negative: Alfalfa mosaic virus (Alfamovirus), Citrus leprosis virus C (Cilevirus), Hibiscus chlorotic ringspot virus (Carmovirus), Hibiscus green spot virus (Higrevirus),
Hibiscus latent Singapore virus, Hibiscus latent Fort Pierce virus (both Tobamovirus) and Tobacco streak virus (Ilarvirus). The presented data suggests that the EMDV isolate in this study may represent a novel strain, and the symptoms induced by this EMDV strain may be untypical. In Italy and Spain, likewise, $H$. rosa-sinensis plants in commercial markets were sometimes unnoticeably infected by EMDV, before distributing and planting in public or home gardens (De Stradis et al. 2008; Parrella et al. 2013). These pose quarantine concerns as EMDV may escape from the inspection if imported plants do not express typical symptoms of virus infection.

EMDV has previously been found in Europe (mainly the Mediterranean coast) and some Asian and African countries (CABI/EPPO 2008). To our knowledge, this is the first report of EMDV in Australia.

\section{References}

Babaie G, Izadpanah K (2002) Host range, distribution, isolates and transmission trials of eggplant mottled dwarf virus. Iran J Plant Pathol 38:235-250

CABI/EPPO (2008) Eggplant mottled dwarf virus. Distribution Maps of Plant Diseases April (Edition 1). Wallingford, UK. CABI Map 1019

De Stradis A, Parrella G, Vovlas C, Ragozzino A (2008) Vein yellowing of Hibiscus rosa- sinensis caused by Eggplant mottled dwarf virus in southern Italy. J Plant Pathol 90:359-361

Lockhart BEL (1987) Evidence for identity of plant rhabdoviruses causing vein-yellowing diseases of tomato and Hibiscus rosa-sinensis. Plant Dis 71:731-733

Martelli GP (1969) Bacilliform particles associated with mottled dwarf of eggplant (Solanum melongena L.). J Gen Virol 5:319-320

Martelli GP, Russo M, Rubino L (2011) Eggplant mottled dwarf virus CMI/AAB Descriptions of Plant Viruses no. 421. http://www. dpvweb.net/dpv/showdpv.php?dpvno $=421$

Parrella G, De Stradis A, Greco B, Villanueva F, Fortes IM, NavasCastillo J (2013) First report of Eggplant mottled dwarf virus in China rose in southern Spain. Span J Agric Res 11:204-207

Plavsic B, Milicic D (1984) Rhabdovirus-like particles associated with vein yellowing of Hibiscus rosa-sinensis L. Acta Horticult 164:4143

Roggero P, Milne RG, Masenga V, Ogliara P, Stravato VM (1995) First reports of eggplant mottled dwarf rhabdovirus in cucumber and in pepper. Plant Dis 79:321

Tang J, Ward LI, Clover GRG (2013) The diversity of strawberry latent ringspot virus in New Zealand. Plant Dis 97:662-667 